\title{
Molecular characterization and application of a novel cytoplasmic male sterility-associated mitochondrial sequence in rice
}

\author{
Yanping Tan ${ }^{1}$, Xin Xu' ${ }^{1}$ Chuntai Wang ${ }^{1}$, Gang Cheng ${ }^{1}$, Shaoqing Li ${ }^{2}$ and Xuequn Liu*
}

\begin{abstract}
Background: Cytoplasmic male sterility (CMS) is a maternally inherited inability to produce functional pollen found in numerous flowering plant species. CMS is associated with mitochondrial DNA mutation, novel chimeric open reading frames (ORFs), and rearrangement of coding and noncoding regions of the mitochondrial genome.

Results: BLAST (Basic Local Alignment Search Tool) analysis indicated that L-sp1, a new sequence-characterized amplified region, is non-homologous to atp6-orfH79 (or atp6-orf79) and WA352 cloned CMS-associated genes. L-sp1 was found in 11 of 102 wild rice accessions belonging to four AA genome species: Oryza rufipogon, Oryza nivara, Oryza glumaepatula, and Oryza meridionalis. Using L-sp1, two new CMS lines were developed, from either low natural fertility plants or sterile plants, by backcrossing $\mathrm{BC}_{1} \mathrm{~F}_{1}$ with Yuetai B. Northern blot and RT-PCR revealed that L-sp1 was only expressed in the anthers of w1/YTB, w2/YTB, w1/YTB//YTB, and w2/YTB//YTB when in the same cytoplasm background.

Conclusions: L-sp1 is a single-copy chimeric CMS-associated gene found in the mitochondrial genome. It can be expressed in anthers with the same specific cytoplasm background, and will be a useful molecular marker for the development and marker-assisted selection of new CMS lines.
\end{abstract}

Keywords: Chimeric DNA L-sp1, Cytoplasmic male sterility, Mitochondrial genome, Rice

\section{Background}

Cytoplasmic male sterility (CMS) is found in numerous flowering plant species. It is maternally inherited, and causes production of non-functional pollen [1]. In many cases, rearrangement of mitochondrial DNA generates novel chimeric open reading frames (ORFs), resulting in expression of novel polypeptides. Chimeric ORFs are derived from coding and noncoding regions, and are located adjacent to genes coding normal mitochondrial function. The rearrangement of mitochondrial DNA can result in deletion and non-functionality of these mitochondrial genes [2]. Over 50 mitochondrial genes have been identified in various plant species [3-6]. In many species, male fertility can be restored using a nuclear fertility restorer $(R f)$ gene. The CMS/Rf system is widely

\footnotetext{
* Correspondence: xqliuw@mail.scuec.edu.cn

${ }^{1}$ Hubei Provincial Key Laboratory for Protection and Application of Special Plants in Wuling Area of China, College of Life Science, South-Central University for Nationalities, Wuhan 430074, China

Full list of author information is available at the end of the article
}

used in the production of hybrid seeds as it eliminates the need for hand emasculation. Moreover, it makes an excellent model system for studying interactions between nuclear and mitochondrial genomes.

Rice (Oryza sativa L.) is an important crop, providing a major food source for about half of the world's population. Since the discovery of the first CMS line [7], over 60 CMS lines, from various origins, have been developed from inter-species, inter-subspecies, and inter-varieties of Oryza plants with the AA genome [8]. CMS lines of rice are mainly divided into wild-abortive (WA), Honglian (HL), and Boro II (BT) groups according to distinctive cytological and genetic characteristics [9]. The WA-CMS system was broadly used for hybrid rice production in China by the end of the 20th century. Its irregularly shaped pollen aborts at the uninucleate stage with negative stainability in $1 \% \mathrm{I}_{2}-\mathrm{KI}$ solution. Recent studies suggest that the mitochondrial gene, WA352, confers WA-CMS by interacting with the nuclear-encoded mitochondrial protein COX11. WA352-induced sterility can be 
suppressed using two restorer-of-fertility (Rf) genes [10]. BT-CMS is the most fully characterized CMS system in rice. Its pollen aborts at the trinucleate stage and is partly stainable in $1 \% \mathrm{I}_{2}$-KI solution, revealing black spherical pollen grains. An unusual chimeric sequence called orf79 encodes a cytotoxic peptide in the mitochondrial genome of the BT-CMS line [11]. This chimeric sequence includes a small portion of the $\operatorname{cox} 1$ gene, and a sequence of unknown origin [12]. As a new germplasm source, HL-CMS has shown great potential for both hybrid rice production and nucleo-cytoplasmic interaction studies. Its spherically shaped pollen aborts at the dinucleate stage with negative stainability in $1 \% \mathrm{I}_{2}-\mathrm{KI}$ solution. Previous studies revealed that coexpression of atp6-orfH79 might interfere with construction of $\mathrm{F}_{0} \mathrm{~F}_{1}$-ATPase during microgenesis $[13,14]$. Recent studies have focused on the chimeric gene orfH79 in the HL-CMS line. This chimeric gene shows 97\% similarity to orf79, and has a 6-base pair (bp) addition at the intercistronic linker between H-atp6 and orfH79 that is absent in orf79. It also encodes a cytotoxic peptide, and affects the development of male gametophytes and the roots $[15,16]$.

Over $17 R f$ or loci for different CMS systems have been reported in rice [11,17-24]. With regard to the $\mathrm{HL}-\mathrm{CMS} / R f$ system, $R f 5$ has been finely mapped on chromosome 10 [21]. It encodes a pentatricopeptide repeat protein, and it physically interacts with GRP162, a Gly-rich protein encoding 162 amino acids, to form a restoration of fertility complex that cleaves CMS-associated transcripts and restores fertility [25]. Another Rf6 gene has been mapped to a region of approximately $200 \mathrm{~kb}$ between markers RM3710 and RM22242 on the short arm of chromosome 8 [26]. Presently, three alleles or loci for HL-CMS have been identified in wild rice by genetic and allelic analyses [27]. A synergistic relationship exists between CMS and fertility-restoration-related genes in Oryza species [28], and the $R f$ allele interacts with CMS factors in a gene-for-gene manner [29-32]. Thus, other CMS-associated DNA sequences or factors are likely to occur in the HL-CMS/Rf system. In this study, we developed a novel sequence-characterized amplified region (SCAR) marker for L-sp1, a chimeric mitochondrial genomic DNA sequence, using random amplification of polymorphic DNA (RAPD) in mitochondrial genomic DNA. Furthermore, L-sp1 can be used for the development of new CMS lines and identification of new cytoplasmic backgrounds through marker-assisted selection (MAS).

\section{Results}

\section{Development and genetic analysis of SCAR marker}

PCR amplification was performed using total genomic DNA of 28 accessions (Table 1) with 264 random primers (10 nucleotides). A 2100-bp product, named U-18/2100, was amplified when using the RAPD primer OPN U-18
Table 1 The three CMS rice types and $F_{1}$ offspring used in this study

\begin{tabular}{|c|c|c|c|}
\hline No. & Name (Code) & Character & $\begin{array}{l}\text { Type of CMS/Rf } \\
\text { system }\end{array}$ \\
\hline 1 & Yue Tai A, YTA & Sterile line & \multirow[t]{8}{*}{ HL-CMS/Rf system } \\
\hline 2 & Yue Tai B, YTB & $\begin{array}{l}\text { Maintainer } \\
\text { line }\end{array}$ & \\
\hline 3 & YTA/9311, HL-2 & $F_{1}$ hybrid & \\
\hline 4 & 9311 & Restorer line & \\
\hline 5 & $\begin{array}{l}\text { Cong Guang 41A, CG- } \\
41 \mathrm{~A}\end{array}$ & Sterile line & \\
\hline 6 & $\begin{array}{l}\text { Cong Guang 41B, CG- } \\
41 B\end{array}$ & $\begin{array}{l}\text { Maintainer } \\
\text { line }\end{array}$ & \\
\hline 7 & CG-41A/MY23, C-M23 & $F_{1}$ hybrid & \\
\hline 8 & MiYang23, MY23 & Restorer line & \\
\hline 9 & Zhen Shan $97 \mathrm{~A}$, ZSA & Sterile line & \multirow[t]{8}{*}{ WA-CMS/Rf system } \\
\hline 10 & Zhen Shan 97 B, ZSB & $\begin{array}{l}\text { Maintainer } \\
\text { line }\end{array}$ & \\
\hline 11 & ShanYou 63, SY63 & $F_{1}$ hybrid & \\
\hline 12 & Ming Hui 63, MH63 & Restorer line & \\
\hline 13 & Maxie $A, M X A$ & Sterile line & \\
\hline 14 & Maxie B, MXB & $\begin{array}{l}\text { Maintainer } \\
\text { line }\end{array}$ & \\
\hline 15 & Maxie 63, MX63 & $F_{1}$ hybrid & \\
\hline 16 & Ming Hui 63, MH63 & Restorer line & \\
\hline 17 & Liu Qian Xin A, QXA & Sterile line & \multirow[t]{6}{*}{ BT-CMS/Rf system } \\
\hline 18 & Liu Qian Xin B, QXB & $\begin{array}{l}\text { Maintainer } \\
\text { line }\end{array}$ & \\
\hline 19 & HP121, HP121 & Restorer line & \\
\hline 20 & ShiJin A, SJA & Sterile line & \\
\hline 21 & ShiJin B, SJB & $\begin{array}{l}\text { Maintainer } \\
\text { line }\end{array}$ & \\
\hline 22 & PeiC311, PC311 & Restorer line & \\
\hline 23 & YTA/SJB, YAS & $\mathrm{BC}_{7} \mathrm{~F}_{1}$ & \multirow{6}{*}{$\begin{array}{l}\text { Different CMS lines } \\
\text { with the same } \\
\text { SJB nucleus }\end{array}$} \\
\hline 24 & ZSA/SJB, ZAS & $\mathrm{BC}_{7} \mathrm{~F}_{1}$ & \\
\hline 25 & V20A/SJB, VAS & $\mathrm{BC}_{7} \mathrm{~F}_{1}$ & \\
\hline 26 & DA/SJB, DAS & $\mathrm{BC}_{7} \mathrm{~F}_{1}$ & \\
\hline 27 & BoA/SJB, BAS & $\mathrm{BC}_{7} \mathrm{~F}_{1}$ & \\
\hline 28 & GangA/SJB, GAS & $\mathrm{BC}_{7} \mathrm{~F}_{1}$ & \\
\hline
\end{tabular}

Note: ZSA, V20A, DA, BoA, and GangA are different CMS lines developed and applied in China. SJB, Shijing B, a maintainer line from Japonica rice.

(5'-GAGGTCCACA-3') with DNA templates from YTA, CG-41A, HL-2, and C-M23 containing HL-type male sterile cytoplasm (Figure 1A). U-18/2100 was recovered, cloned, and sequenced according to TA-cloning protocols. Following U-18/2100 sequence analysis, a SCAR marker was developed and named as L-sp1 (specific primers, H1: 5'-GAGGTCCACATCCTTCAATC-3'; H2: 5'-AGGTCC ACAAACCACTGAAG-3'). The genetic nature of the Lsp1 fragment was determined by PCR using total genomic DNA of plants from two different backcross populations: 


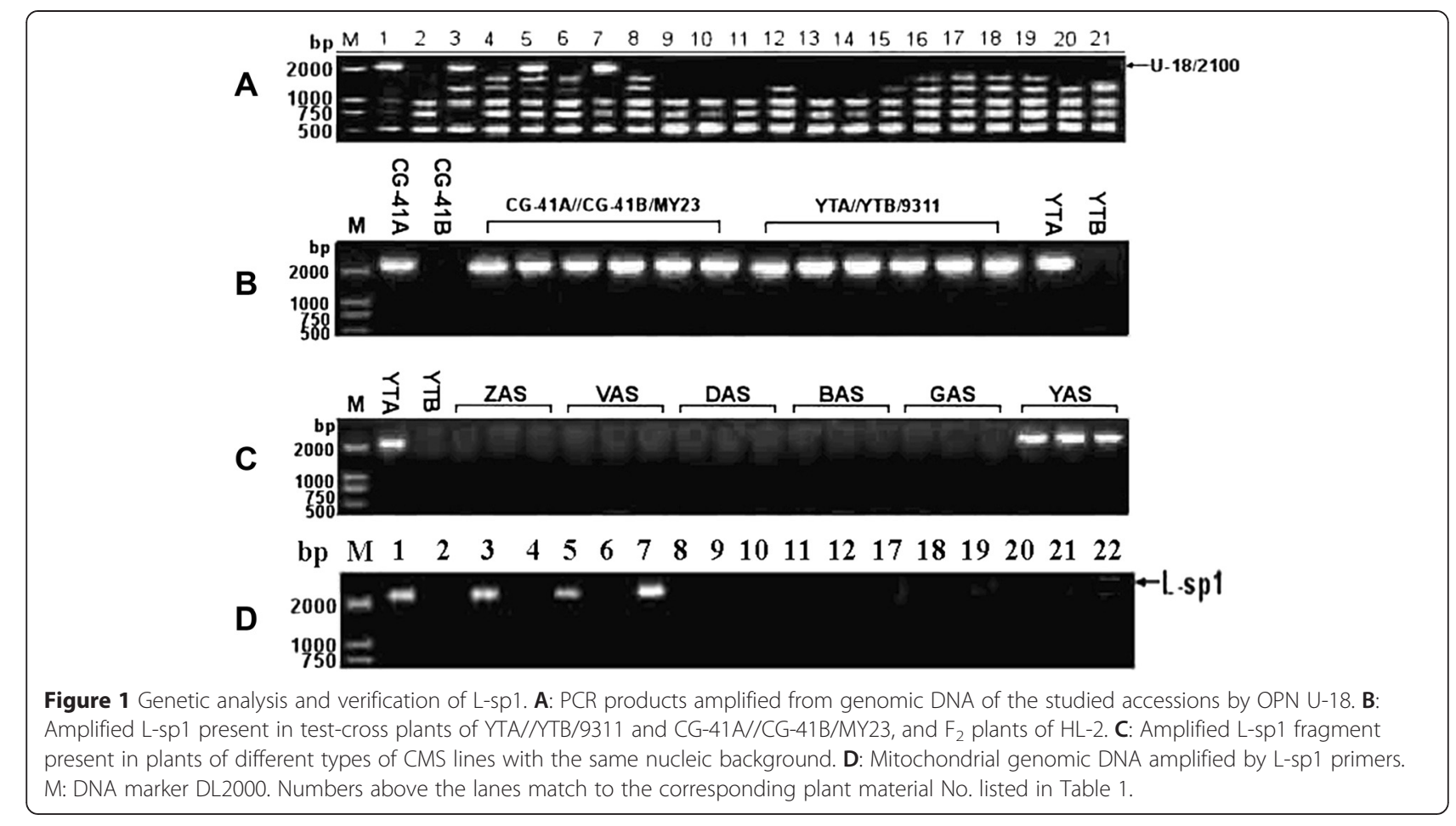

$\mathrm{BC}_{1} \mathrm{~F}_{1} \mathrm{~S}$ CG-41A//CG-41B/MY23 and YTA//YTB/9311. These two backgrounds display HL cytoplasmic male sterility and specific nuclear backgrounds (Figure 1B), as did plants from six different types of CMS lines $\left(\mathrm{BC}_{7} \mathrm{~F}_{1}\right)$ with similar SJB nucleic backgrounds (Figure 1C). The L-sp1 fragment was consistently amplified in all plants possessing the HL cytoplasmic male sterility background, and amplification remained constant with changes in the nucleic genome. L-sp1 can be inherited cytoplasmically or maternally, and has specificity for CMS. Based on characteristics of the cytoplasmic genes and the possible relationship between L-sp1 and CMS, we further verified stability and reliability of the SCAR marker using mitochondrial DNA from 18 out of 28 accessions (Table 1) with specific primers $\mathrm{H} 1$ and $\mathrm{H} 2$. The L-sp1 fragment could be amplified from mitochondrial genomic DNA of YTA, CG-41A, HL-2, and C-M23 (Figure 1D), suggesting that $\mathrm{U}-18 / 2100$ is related to CMS.

Molecular characterization of mitochondrial SCAR marker To assay HL-CMS specificity and L-sp1 copy number, mitochondrial genomic DNA of CG-41A, YTA, HL-2, YAS, YTB, and of all accessions was digested with EcoRI or $B a m \mathrm{HI}$ and hybridized with L-sp1 probe. A single fragment of $23 \mathrm{~kb}$ was found when using L-sp1 (2176 bp specific primer, H1: 5'-GAGGTCCACATCCTTCAAT C-3'; H2: 5'-AGGTCCACAAACCACTGAAG-3') and Natp6 (S59890) probes (F: 5'-CAATCCTTGGTAGAGTG3'; R: 5'-TAATGGCAGTGGGACTCC-3') for all accessions following digestion with $B a m \mathrm{HI}$. The band was the same size in CG-41A, YTA, HL-2, and YAS, but smaller in YTB (Figure 2A and B). Three bands were detected when using the L-sp1 probe following digestion with EcoR1, there were two bands detected in CG-41A, YTA, $\mathrm{HL}-2$, and YAS, and one band in YTB (Figure 2C). One band in YTB, and two bands in CG-41A, YTA, HL-2, and YAS were detected when using the $N$-atp6 probe following digestion with EcoR1 (Figure 2D). These results indicated that L-sp1 was single copy, and could be used as a characteristic molecular marker in the mitochondrial genome.

L-sp1 is a chimeric mitochondrial genomic DNA sequence of $2176 \mathrm{bp}$ (HQ267715). When compared with the mitochondrial genomic DNA sequence, L-sp1 was determined to contain four Japonica rice (Nipponbare) DNA fragments (Figure 3A). Except for a 10-bp gap located between bp 1724 and 1725 , the sequence from bp 1 to 1933 of L-sp1 showed $99 \%$ similarity to bp 224994 to 223054 of the NC_011033.1 clone. The L-sp1 sequence from bp 1684 to 1933 showed 99\% similarity to bp 282437 to 282180 and 413358 to 413001 of the same clone. The L-sp1 sequence from bp 1934 to 2100 showed 99\% similarity to bp 343650 to 343483 and 424737 to 424570 of the NC_011033.1 clone, and 100\% similarity to the cDNA sequence located at 1062 to $1228 \mathrm{bp}$ of the mitochondrial ribosomal protein L5 gene. The remaining 56-bp DNA sequence (bp 2119 to 2174 ) of L-sp1 was similar to bp 181916 to 181862 of the NC_011033.1 clone.

Sequence comparison of sequences between L-sp1 and indica WA-CMS mitochondrial genomic DNA (Figure 3B) 


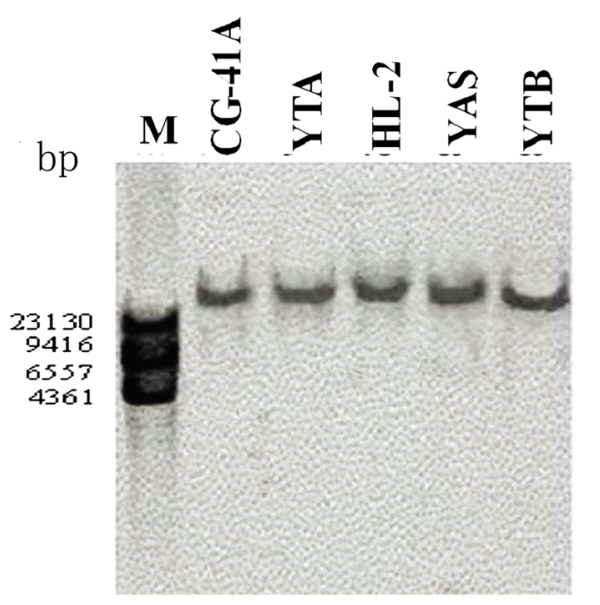

A

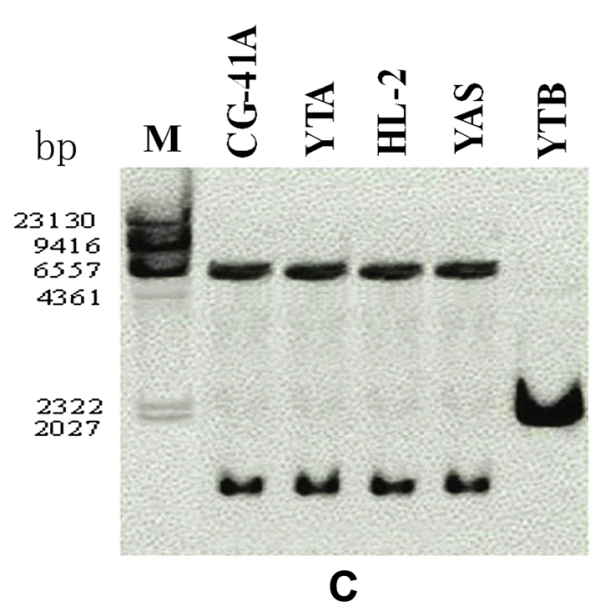

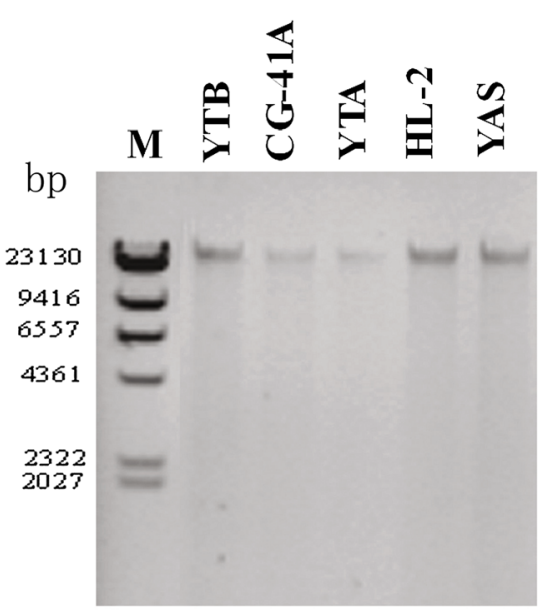

B

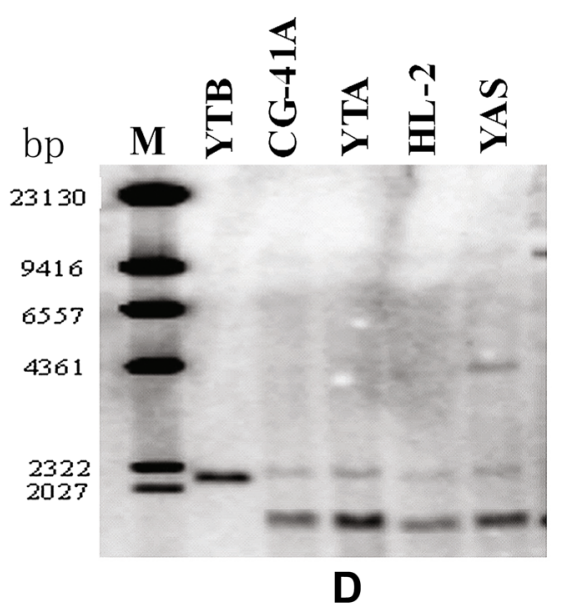

Figure 2 Southern analysis. M: Lambda DNA/Hind III Marker. A, B: Mitochondrial genomic DNA of CG-41A, YTA, HL-2, and YTB, and YAS cut with BamHI. C, D: Mitochondrial genomic DNA of CG-41A, YTA, HL-2, and YTB, and YAS cut with EcoRI. A, C: Southern analysis with L-sp1 probes. B, D: Southern analysis with $\mathrm{N}$-atp6 probes. $\mathrm{L}$-sp1 probe was amplified using the sequence-specific primers $\mathrm{H} 1$ and $\mathrm{H} 2$, these contain one EcoRI restriction site and no BamHI restriction site. $\mathrm{N}$-atp6 probe was amplified using sequence-specific primers $\mathrm{F}$ and $\mathrm{R}$, and also contain one EcoRI restriction site and no BamHI restriction site. A part of L-sp1 probe is homologous to a part of the $\mathrm{N}$-atp6 probe.

was as follows: the L-sp1 sequence from bp 1 to 1722 showed $98 \%$ similarity to bp 349531 to 351252 of JF281154.1; bp 1682 to 1931 showed 97\% similarity to bp 37787 to 37530 and 219740 to 219997 of JF281154.1; and bp 1931 to 2098 of L-sp1 showed 100\% similarity to bp 338549 to 338716 of JF281154.1. Other sequences shared no homology to the indica WA-CMS mitochondrial genome.

BLAST analysis of L-sp1, WA352 (AGG40956), N-atp6, and atp6-orfH79 sequences [25] revealed that L-sp1 sequences from bp 1 to 392 were entirely homologous to the 3' flanking sequence of the ORF of $N$-atp6. Other DNA sequences showed no homology between L-sp1 and $N$-atp6. Furthermore, L-sp1 was non-homologous to the total DNA sequences of both atp6-orfH79 and WA352 (Figure 3C).

\section{Distribution of L-sp1 in the AA genome of wild rice}

To determine the distribution of L-sp1 in the AA genome of wild rice, PCR amplification was performed using L-sp1 sequence-specific primers $\mathrm{H} 1$ and $\mathrm{H} 2$. Bands of approximately $2176 \mathrm{bp}$, the same size as those in YTA, were found in 11 of 102 investigated wild rice accessions (Figure 4A). These 11 accessions belonged to four species: three from O. rufipogon (103423, 105696, 105698), five from O. nivara (101978, 103415, 103835, 105712, 106153), two from O. glumaepatula (100968, 105661), and one from O. meridionalis (82042), these accessions came from Cambodia, India, Sri Lanka, Suriname, Brazil, Bangladesh and Laos in Southeast Asia, West Africa, South America, and Oceania, respectively. To analyze the distribution of orfH79 in the 11 wild rice accessions, PCR was performed using orfH79 sequence-specific primers 

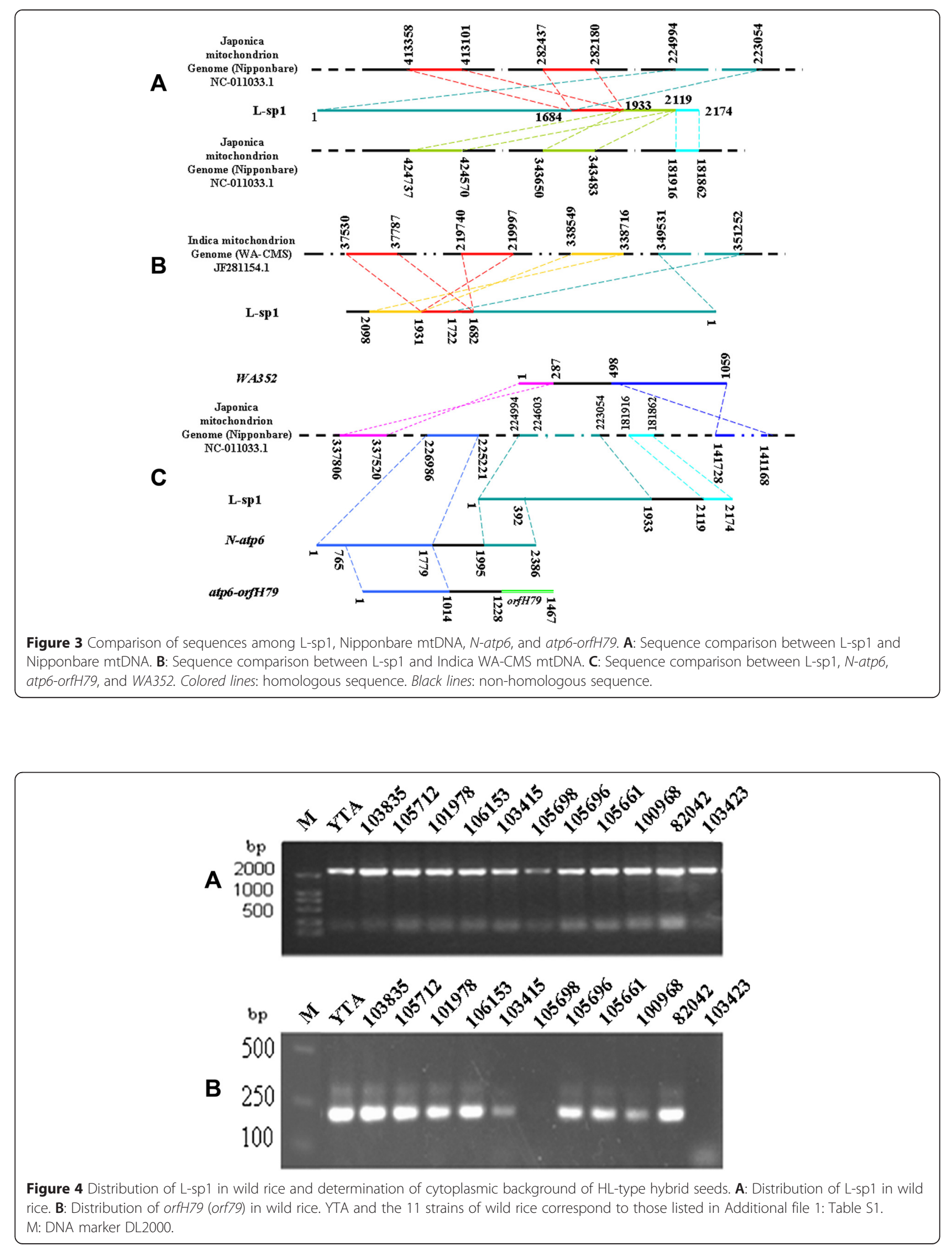
(O1: 5'-ATGACAAATCTGCTCCGAT-3'; O2: 5'-TTAC TTAGGAAAGACTACAC-3'). This revealed that 9 of the 11 wild accessions amplified the same sized band, approximately $240 \mathrm{bp}$, as YTA, while the other two accessions $(103423,105698)$ failed to amplify a PCR product (Figure 4B).

\section{Development of new CMS lines via backcrosses from accessions containing L-sp1}

To validate whether L-sp1 was related to CMS at the molecular genetic level, an interspecies cross was performed using two accessions (103423 and 105698, now named w1 and w2, respectively) carrying L-sp1 as maternal parents with YTB. Fertility analysis revealed the percentage of stainable pollen grains of the $F_{1}$ hybrids $w 1 / Y T B$ and w2/YTB were $10.3 \% \pm 1.5 \%$ and $15.8 \% \pm 2.3 \%$, respectively; over $50 \%$ of abortive pollen grains were spherical (Figure 5). The seed-setting rates of bagged spikelets for the same crosses were $13.6 \% \pm 1.5 \%$ and $23.5 \% \pm 2.5 \%$, respectively (Table 2). To elucidate whether male sterility of the testcross derived from potential incompatibility between species or subspecies, the HL maintainer YTB was crossed as a female parent with w1 and w2. Fertility assessment revealed that $\mathrm{YTB} / \mathrm{w} 1$ and $\mathrm{YTB} / \mathrm{w} 2$ were both fertile $(\sim 80 \%$ pollen fertility; $\sim 50 \%$ seed-setting fertility). This indicated that the fertility of crosses between wild rice and YTB was mainly influenced by the cytoplasm genome as opposed to the nuclear genome in wild rice. Next, fertility of populations derived from $\mathrm{BC}_{1} \mathrm{~F}_{1}$ backcrosses of $\mathrm{w} 1 / \mathrm{YTB} / / \mathrm{YTB}$ and $\mathrm{w} 2 / \mathrm{YTB} / / \mathrm{YTB}$ were examined; the spherical abortive grain rate increased from $\sim 25 \%$ to $\sim 85 \%$, and fertility of pollen and seed-setting was clearly reduced (Figure 5). Therefore, two new CMS lines could be developed from low fertility and sterile plants belonging to $\mathrm{BC}_{1} \mathrm{~F}_{1}$ (w1/YTB//YTB and $w 2 / Y T B / / Y T B)$ or $\mathrm{BCnF}_{1}$ (n, generation number of backcross) maternal parents, by successive backcrossing with YTB.

Genetic and transcript analysis of L-sp1 in new CMS lines PCR amplifications were performed using mtDNA from YTA, YTB, w1/YTB, w2/YTB, YTB/w1, YTB/w2, w1/ $\mathrm{YTB} / / \mathrm{YTB}$, and $\mathrm{w} 2 / \mathrm{YTB} / / \mathrm{YTB}$ plants. When using L-sp1 specific primers $\mathrm{H} 1$ and $\mathrm{H} 2$, an L-sp1 amplicon was observed in plants with the same cytoplasmic background as w1 and w2 (w1/YTB, w2/YTB, w1/YTB//YTB, and w2/ $\mathrm{YTB} / / \mathrm{YTB}$ ), while the L-sp1 amplicon was not amplified from plants with a different cytoplasm background than w1 and w2 (YTB, YTB/w1, and YTB/w2). This revealed

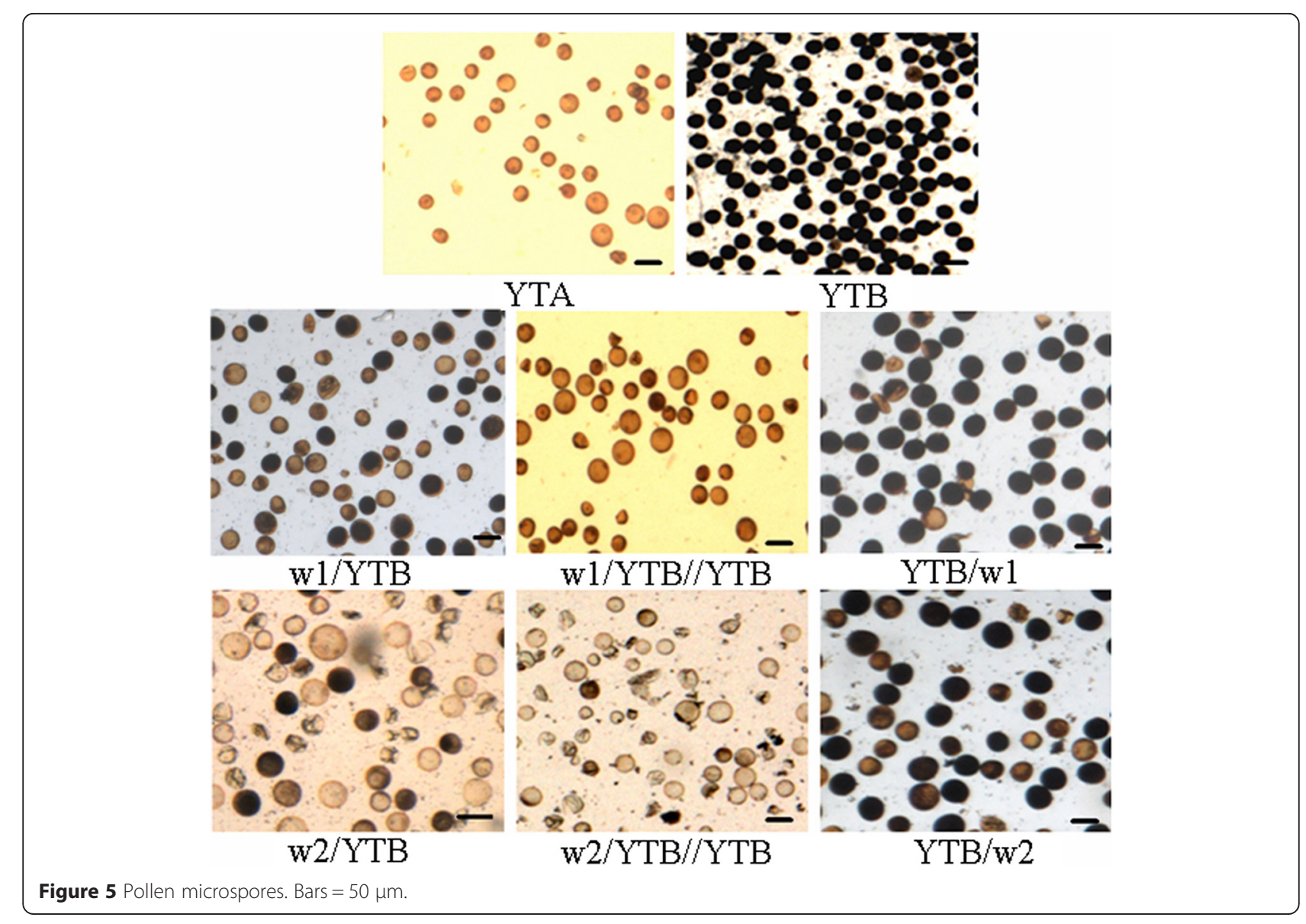


Table 2 Fertility analysis of hybrid $F_{1}$ and $B C_{1} F_{1}$ backcrossed lines

\begin{tabular}{|c|c|c|c|c|c|c|}
\hline \multirow{2}{*}{$\begin{array}{l}\text { Accessions } \\
\text { Combination }\end{array}$} & \multicolumn{3}{|c|}{103423 (w1) \% } & \multicolumn{3}{|c|}{105698 (w2) \% } \\
\hline & w1/YTB & YTB/w1 & w1/YTB//YTB & w2/YTB & YTB/W2 & w2/YTB/NTB \\
\hline Spherical abortive & $54.3 \pm 2.6$ & & $82.5 \pm 2.8$ & $61.2 \pm 3.4$ & & $86.5 \pm 2.1$ \\
\hline Typical abortive & $35.4 \pm 1.8$ & & $13.0 \pm 2.1$ & $23.0 \pm 4.5$ & & $10.3 \pm 2.7$ \\
\hline Pollen fertility & $10.3 \pm 1.5$ & $77.3 \pm 3.2$ & $4.5 \pm 0.6$ & $15.8 \pm 2.3$ & $82.3 \pm 1.9$ & $3.2 \pm 0.4$ \\
\hline Seed-setting fertility & $13.6 \pm 1.5$ & $45.6 \pm 2.4$ & $7.8 \pm 1.1$ & $23.5 \pm 2.5$ & $54.2 \pm 2.2$ & $3.5 \pm 2.1$ \\
\hline
\end{tabular}

that L-sp1 showed cytoplasmic or maternal inheritance. In addition, the HL-CMS gene orfH79 was not amplified from the above plants (with the exception of YTA) when using the orfH79 primer set (O1 and O2) (Figure 6A).

To examine the expression manner of L-sp1 in the $F_{1}$ and backcrossed $\mathrm{BC}_{1} \mathrm{~F}_{1} \mathrm{w} 1$ and $\mathrm{w} 2$ backgrounds, RTPCR was performed using total RNA from rice anthers with L-sp1 as probe. L-sp1 was found to be expressed in the anthers of w1/YTB, w2/YTB, w1/YTB//YTB, and $\mathrm{w} 2 / \mathrm{YTB} / / \mathrm{YTB}$ in a similar manner to YTA (Figure 6B).

\section{Discussion}

The DNA sequence analysis results suggest no homologous sequence exists between L-sp1 and orfH79 or atp6-orfH79. In wild rice, 19 of 102 investigated wild rice accessions could amplify PCR products of $\sim 240$ bp when using orfH79 sequence-specific primers $\mathrm{O} 1$ and $\mathrm{O} 2$, a similar product was amplified from YTA. Sequencing of these 19 PCR fragments revealed that eight of the DNA sequences had the same single-nucleotide polymorphism (SNP) as orf79 in BT-CMS [12], while the remaining 11 accessions had the same SNP as orfH79 in HL-CMS [33]. In addition, a 2176-bp fragment amplified from L-sp1 specific primers was found present in 11 of 102 wild rice accessions. Nine of these eleven accessions (82042, 101978, 103415, 103835, 105712, 106153, 106321, 100968, and 105661) contain both orfH79 and L-sp1 sequences, while the other two accessions $(103423,105698)$ contain only L-sp1. Previous studies documented four completely sterile alloplasmic CMS lines (w15A, w20A, w34A, w46A), developed from w15 (101971), w20 (103836), w34 (105419), and w46 (106321) by successive recurrent backcrossing of sterile plants from a $\mathrm{BC}_{1} \mathrm{~F}_{1}$ population with the $\mathrm{HL}$ maintainer YTB, respectively [34]. Using the same method, two nearly sterile CMS lines were developed from w1 and w2 accessions carrying L-sp1.

Recent studies revealed CMS-associated mitotypes are compatible with $R f$-candidate-related nucleotypes, and CMS and $R f$ have a parallel evolutionary relationship in Oryza [28]. Several studies suggest that different $R f$ alleles interact with CMS in a gene-for-gene manner. Therefore, various $R f$ loci are determined by the multiple

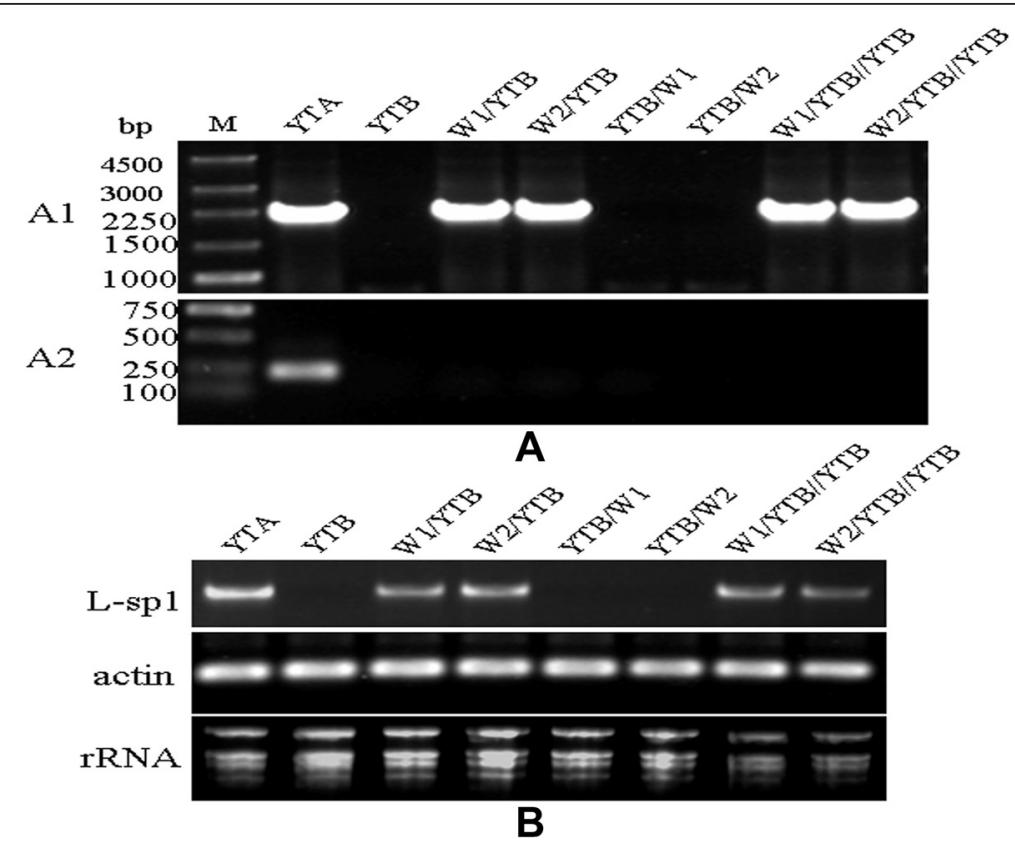

Figure 6 Genetic and transcript analysis of L-sp1 in the newly developed CMS lines. A: Genetic analysis: A1, Genetic pattern of L-sp1; A2, Genetic pattern of orfH79. B: Transcript analysis. M: DNA markers: 250-bp DNA ladder/DL2000. w1: accession 103423; w2: accession 105698. 
CMS systems existing in the natural populations within plant species [29-32]. We plan to further analyze the restoration and maintenance relationship, and the fertility restoring model of the two CMS lines developed in this study.

\section{Conclusions}

L-sp1 is a 2176-bp CMS-associated chimeric and singlecopy DNA fragment found in the mitochondrial genome. It could be expressed in the anthers of all low natural fertility plants or sterile plants with a similar cytoplasm background. Therefore, L-sp1 can be used for both identification of the cytoplasmic background in marker-assisted selection programs and in the development of new CMS lines.

\section{Methods}

\section{Plant materials}

One hundred and two accessions of AA-genome wild rice were obtained from the International Rice Research Institute (IRRI; Los Baños, Laguna, Philippines; Additional file 1: Table S1). These included 22 parent plants, $\mathrm{F}_{1}$ hybrids obtained from three types of CMS/Rf system (HL-, WA-, and BT-type), and six other CMS lines, each of which had different cytoplasms but identical Shijing B (SJB, a Japonica rice maintainer line) nucleus backgrounds (Table 1). All plant materials were planted in the experimental field within the South-Central University for Nationalities campus in Wuhan, Hubei province in China during summers and in Lingshui, Hainan province in China during winters of 2009 to 2013.

\section{Isolation of mitochondrial DNA and nuclear DNA}

To isolate mtDNA, $20 \mathrm{~g}$ of young leaves were harvested using the modified method reported by Yi et al. [33], following etiolation they were homogenized in $80 \mathrm{~mL}$ of homogenizing buffer (pH7.2) containing 0.4 M mannitol, $40 \mathrm{mM}$ MOPS, $1 \mathrm{mM}$ EDTA, 0.05\% cysteine, 0.1\% BSA and $0.03 \%$ mercaptoethonal. After differential centrifugation and DNase I processing (Promega, USA), the pellet was resuspended with lysis buffer and fixed at room temperature for $5 \mathrm{~min}$. Following phenol-chloroform extraction, DNA was precipitated with ethanol.

Total nuclear genomic DNA was isolated from green leaves using the modified method described by Zhang et al. [35]. DNA quality and quantity were estimated spectrophotometrically using a specific amount of lambda DNA (MBI, USA) on an agarose gel, and by visualizing under ultraviolet light.

\section{Polymerase chain reaction amplification}

DNA amplification was performed in a programmable thermal controller (PTC-100, MJ Research, USA) using the following program: $5 \mathrm{~min}$ at $94^{\circ} \mathrm{C}$; 35 cycles of $1 \mathrm{~min}$ at $94^{\circ} \mathrm{C}, 1 \mathrm{~min}$ at $38^{\circ} \mathrm{C}$ (RAPD analysis) or $58^{\circ} \mathrm{C}$ (SCAR analysis), $1.5 \mathrm{~min}$ at $72^{\circ} \mathrm{C}$; 8 -min final extension at $72^{\circ} \mathrm{C}$. PCRs were performed in a $25-\mu \mathrm{L}$ reaction volume (10 mM Tris- $\mathrm{HCl}(\mathrm{pH} 8.8), 25 \mathrm{mM} \mathrm{KCl}, 1.5 \mathrm{mM}$ $\mathrm{MgCl}_{2}, 0.8 \mathrm{mM} \mathrm{dNTPs}, 0.2 \mathrm{mM}$ random primer (10 nucleotides), 100 ng genomic DNA, and 1 unit of Taq polymerase (Takara, Japan). Amplified RAPD fragments were electrophoretically separated using 1.5\% agarose gels, stained with ethidium bromide, and photographed under ultraviolet light using Gel Doc2000 (Bio-Rad, USA). Molecular weights were estimated using a molecular marker (DL2000, Takara).

\section{DNA sequencing and SCAR development}

DNA polymorphism bands of RAPD markers were isolated and collected from agarose gels using a PCR purification kit according to manufacturer's specifications (MBI). Purified PCR products were cloned using a pGEMT easy system I kit (Promega, USA) and sequenced by Shanghai Sangon Biological Engineering Technology and Services (Shanghai, China). The polymorphism sequences were used to design new primers and develop SCAR markers linked to different CMS types. The newly developed primers were used to amplify mitochondrial DNA from all materials mentioned in this study.

\section{Southern hybridization}

L-sp1 (2176 bp) and N-atp6 probes were amplified using primers based on corresponding mitochondrial genomic sequences of rice. Mitochondrial DNA $(20 \mu \mathrm{g})$ was separated on $0.8 \%$ agarose gels following digestion with either EcoRI or BamHI (New England Biolabs, USA) and transferred to Hybond $\mathrm{N}^{+}$-nylon membranes. Probes were radioactively labeled by random priming with $\alpha-{ }^{32} \mathrm{P}-\mathrm{dCTP}$. Southern hybridization was performed in hybridization buffer at $65^{\circ} \mathrm{C}$ for $16 \mathrm{~h}$. The membrane was washed twice at room temperature for $15 \mathrm{~min}$ with $2 \times$ SSC containing $0.1 \%$ sodium dodecyl sulfate (SDS) and at $60^{\circ} \mathrm{C}$ for 30 min with $0.1 \times \mathrm{SSC}$ containing $0.1 \%$ SDS, and was then autoradiographed.

\section{Transcript analysis}

Total RNA was isolated from rice anthers using TRIzol reagent according to the manufacturer's instructions (Invitrogen, USA). It was then extracted with chloroform, precipitated in isopropyl alcohol, and rinsed with ethanol before being dissolved in RNase-free water. RNase-Free DNase I (Promega, USA)was added to remove any possible genomic DNA contaminants. Synthesis of first-strand cDNA was obtained from the total RNA using a cDNA Synthesis Kit (Toyobo, Japan). The RT-PCR reaction was terminated after 22 cycles, and rice actin was used as a control. All assays were repeated at least three times. 


\section{Additional file}

Additional file 1: Table S1. All wild rice in the study.

\section{Abbreviations}

CMS: Cytoplasmic male sterility; ORFs: Open reading frames; BLAST: Basic Local Alignment Search Tool; YTA: Yuetai A; YTB: Yuetai B; Rf: Fertility restorer; WA: Wild-abortive; HL: Honglian; BT: Boro II; SCAR: Sequence-characterized amplified region; RAPD: Random amplification of polymorphic DNA MAS: Marker-assisted selection; IRRI: International Rice Research Institute SJB: Shijing B; bp: Base pairs; DNase: Deoxyribonuclease; RNase: Ribonuclease; dNTP: Deoxynucleotide-5/-triphosphate; EDTA: Ethylenediamine tetraacetic acid; MOPS: 3- (N-Morpholino) propanesulfonic acid; BSA: Bovine Serum Albumin; PCR: Polymerase chain reaction.

\section{Competing interests}

The authors declare that they have no competing interests.

\section{Authors' contributions}

YP Tan and XQ Liu designed the research experiments. YP Tan, X Xu, CT Wang, G Cheng, SQ Li, and XQ Liu performed the experimental research. YP Tan, X Xu, and XQ Liu analyzed the data. YP Tan and XQ Liu wrote and prepared the manuscript. All authors read and approved the final manuscript.

\section{Acknowledgments}

We thank IRRI for kindly providing wild rice. This work was supported by the National Natural Science Foundation of China (Grant Nos. 30771163 and 31170296), and the Academic Innovation Team for Plant Development and Genetics at the South-Central University for Nationalities (XQ Liu group)

\section{Author details}

${ }^{1}$ Hubei Provincial Key Laboratory for Protection and Application of Special Plants in Wuling Area of China, College of Life Science, South-Central University for Nationalities, Wuhan 430074, China. ${ }^{2}$ Key Laboratory of MOE for Plant Developmental Biology, College of Life Science, Wuhan University, Wuhan 430072, China.

\section{Received: 25 November 2014 Accepted: 22 April 2015}

\section{Published online: 30 April 2015}

\section{References}

1. Wise RP, Pring DR. Nuclear-mediated mitochondrial gene regulation and male fertility in higher plants: light at the end of the tunnel? Proc Natl Acad Sci U S A. 2002;99:10240-2.

2. Chase CD, Babay-Laughnan S. Cytoplasmic male sterility and fertility restoration by nuclear genes. In: Daniell H Chase CD, editors. Molecular Biology and Biotechnology of Plant Organelles. Kluwer Academic publisher: Dordrecht; 2004. 593-622

3. Unseld M, Marienfeld JR, Brandt P, Brennicke A. The mitochondrial genome of Arabidopsis thaliana contains 57 genes in 366,924 nucleotides. Nat Genet. 1997:15:57-61.

4. Kubo T, Nishizawa S, Sugawara A, Itchoda N, Estiati A, Mikami T. The complete nucleotide sequence of the mitochondrial genome of sugar beet (Beta vulgaris L.) reveals a novel gene for tRNACys (GCA). Nucleic Acids Res. 2000;28:2571-6.

5. Notsu Y, Masood S, Nishikawa T, Kubo N, Akiduki G, Nakazono M, et al. The complete sequence of the rice (Oryza sativa L.) mitochondrial genome: frequent DNA sequence acquisition and loss during the evolution of flowering plants. Mol Genet Genomics. 2002;268:434-45.

6. Sugiyama $Y$, Watase $Y$, Nagase $M$. The complete nucleotide sequence and multipartite organization of the tobacco mitochondrial genome: comparative analysis of mitochondrial genomes in higher plants. Mol Genet Genomics. 2005;272:603-15.

7. Shinjyo C. Cytoplasmic-genetic male sterility in cultivated rice, Oryza sativa L. II: the inheritance of male sterility. Jpn J Genet. 1969;44:149-56.

8. Li JM, Yuan LP. Hybrid rice: genetics, breeding, and seed production. Plant Breeding Rev. 2000;17:15-158.

9. Zhu YG. Studies on rice male sterility and its restoration. In: Biology of male sterility in rice. Wuhan Univ Press: Wuhan; 2000. p. 12-3.
10. Luo DP, Xu H, Liu ZL, Guo JX, Li HY, Chen LT, et al. A detrimental mitochondrial-nuclear interaction causes cytoplasmic male sterility in rice. Nat Genet. 2013;45:573-7.

11. Wang ZH, Zou YJ, Li XY, Zhang QY, Chen LT, Wu H, et al. Cytoplasmic male sterility of rice with Boro II cytoplasm is caused by a cytotoxic peptide and is restored by two related PPR motif genes via distinct modes of mRNA silencing. Plant Cell. 2006;18:676-87.

12. Akagi $H$, Sakamoto $M$, Shinjyo $C$, Shimada $H$, Fujimura $T$. A unique sequence located downstream from the rice mitochondrial atp6 may cause malesterility. Curr Genet. 1994;25:52-8.

13. Zhang H, Li S, Yi P, Wan C, Chen Z, Zhu Y. A Honglian CMS line of rice displays aberrant $F_{0}$ of $F_{0} F_{1}$-ATPase. Plant Cell Rep. 2007;26:1065-71.

14. Wan C, Li S, Wen L, Kong J, Wang K, Zhu Y. Damage of oxidative stress on mitochondria during microspores development in Honglian CMS line of rice. Plant Cell Rep. 2007;26:373-82.

15. Peng XJ, Li FH, Li SQ, Zhu YG. Expression of a mitochondrial gene orfH79 from the CMS-HongLian rice inhibits saccharomyces cerevisiae growth and causes excessive ROS accumulation and decrease in ATP. Biotechnol Lett. 2009:31:409-14.

16. Peng XJ, Wang K, Hu CF, Zhu YL, Wang T, Yang J, et al. The mitochondrial gene orfH79 plays a critical role in impairing both male gametophyte development and root growth in CMS-Honglian rice. BMC Plant Biol. 2010;10:125-35

17. Yao FY, Xu CG, Yu SB, Li JX, Gao YJ, Li XH, et al. Mapping and genetic analysis of two fertility restorer loci in wild-abortive cytoplasmic male sterility system of rice (Oryza sativa L.). Euphytica. 1997;98:183-7.

18. Xie JK, Zuang JY, Fan YY, Tu GQ, Xia YW, Zheng KL. Mapping of fertility restoring genes with main effects and epistatic effects for CMS-DA in rice. Acta Genetica Sinica. 2002;29:565-70.

19. Komori T, Yamamoto T, Takemori N, Kashihara M, Matsushima H, Nitta N. Fine genetic mapping of the nuclear gene, Rf-1, that restores the BT-type cytoplasmic male sterility in rice (Oryza sativa L.) by PCR-based markers. Euphytica. 2003;129:241-7.

20. Akagi H, Nakamura A, Yokozeki-Misono Y, Inagaki A, Takahashi H, Mori K, et al. Positional cloning of the rice Rf-1 gene, a restorer of BT-type cytoplasmic male sterility that encodes a mitochondria-targeting PPR protein. Theor Appl Genet. 2004;108:1449-57.

21. Liu XQ, Xu X, Tan YP, Li SO, Hu J, Huang JY, et al. Inheritance and molecular mapping of two fertility-restoring loci for Honglian gametophytic cytoplasmic male sterility in rice (Oryza sativa L.). Mol Genet Genomics. 2004;271:586-94.

22. Li SQ, Yang DC, Zhu YG. Characterization and use of male sterility in rice breeding. J Integr Plant Biol. 2007;49:791-804.

23. Fuji S, Toriyama K. Suppressed expression of retrograde-regulated male sterility restores pollen fertility in cytoplasmic male sterile rice plants. Proc Nat Acad Sci U S A. 2009;106:9513-8.

24. Itabashi E, Iwata N, Fujii S, Kazama T, Toriyama K. The fertility restorer gene, Rf2, for Lead rice-type cytoplasmic male sterility of rice encodes a mitochondrial glycine-rich protein. Plant J. 2011;65:359-67.

25. Hu J, Wang K, Huang WC, Liu G, Gao Y, Wang JM, et al. The rice pentatricopeptide repeat protein RF5 restores fertility in Hong-Lian cytoplasmic male-sterile lines via a complex with the Glycine-rich protein GRP162. Plant Cell. 2011;24:109-22.

26. Huang WC, Hu J, Yu CC, Huang Q, Wan L, Wang L, et al. Two non-allelic nuclear genes restore fertility in a gametophytic pattern and enhance abiotic stress tolerance in the hybrid rice plant. Theor Appl Genet. 2011:124:799-807.

27. Tan YP, Li SQ, Wang L, Liu G, Hu J, Zhu YG. Genetic analysis of fertility-restore genes in rice. Biol Plantarum. 2008:52(3):469-74.

28. Tan YP, Li SQ, Xie HW, Duan SH, Wang T, Zhu YG. Genetical and molecular analysis reveals a cooperating relationship between cytoplasmic male sterility- and fertility restoration-related genes in Oryza species. Theor Appl Genet. 2011;122:9-19.

29. Manicacci D, Atlan A, Couvet D. Spatial structure of nuclear factors involved in sex determination in the gynodioecious Thymus vulgaris L. J Evol Biol. 1997;10:889-907

30. Charlesworth D, Laporte $\mathrm{V}$. The male-sterility polymorphism of Silene vulgaris: analysis of genetic data from two populations and comparison with Thymus vulgaris. Genetics. 1998;150:1267-82.

31. Taylor DR, Olson MS, McCauley DE. A quantitative genetic analysis of nuclear-cytoplasmic male sterility in structured populations of Silene vulgaris. Genetics. 2001;158:833-41. 
32. Van Damme JMM, Hundscheid MPJ, Ivanovic S, Koelewijn HP. Multiple CMS-restorer gene polymorphism in gynodioecious Plantago coronopus. Heredity. 2004;93:175-81.

33. Yi $P$, Wang $L$, Sun QP, Zhu $Y$ G. Discovery of mitochondrial chimeric gene associated with male sterility of HL-rice. Chinese Sci Bull. 2002;47:744-7.

34. Li SQ, Tan YP, Wang K, Wan CX, Zhu YG. Gametophytically alloplasmic CMS line of rice (Oryza sativa L.) with variant orfH79 haplotype corresponds to specific fertility restorer. Theor Appl Genet. 2008;117:1389-97.

35. Zhang Q, Saghai MMA, Lu T, Shen B. Genetic diversity and differentiation of indica and japonica rice detected by RFLP analysis. Theor Appl Genet. 1992;83:495-9.

\section{Submit your next manuscript to BioMed Central} and take full advantage of:

- Convenient online submission

- Thorough peer review

- No space constraints or color figure charges

- Immediate publication on acceptance

- Inclusion in PubMed, CAS, Scopus and Google Scholar

- Research which is freely available for redistribution 